Ophthalmologica 1970;161:534-542

\title{
Index rerum ad Vol. 161
}

Confecit: C. Loeb-Schiirch

$(B)=$ Buchbesprechungen - Book reviews - Livres nouveaux $(V)=$ Vortrag - Report -

Communication

Abflusswiderstand, v. Acéclidine,

Pilocarpintest Abhandlungen aus dem Gebiete der

Augenheilkunde, 414 (B) Ablatio retinae, v. Akkommodations-

phosphen, Netzhautatrophie,

Silberklemme Abrasion iodée, v. Kälteapplikation Acéclidine; effet d'un collyre

associant $\Gamma$ acéclidine et $\Gamma$ adrénaline

sur la pression oculaire de patients

atteints de glaucome à angle

ouvert, 394 Acuité visuelle, v. Lichtstreifentest,

Sehschärfenbestimmung

Adaptation to darkness, v. Akkom-

modationsphosphen, Behçet-

Uveitis Adénome oxyphile, v. Oncocytoma Adler's textbook of ophthalmology,

417 (B) Adrenaline, v. Acéclidine Affections carotidiennes; les signes

oculaires dans les affections

carotidiennes, 324 (V) Akkommodationsphosphen; die Aus-

dehnung des Akkommodations-

phosphens im Gesichtsfeld, 499 Akridinorange-Fluorochromierung,

v. Bertalanffy-Methode

Index rerum

ad Vol. 161

535

Albinisme oculaire; une association de stigmates d'albinisme oculaire et d'amaurose congénitale de Leber. Rapport clinique, 425

Almanach für Augenheilkunde, 415 (B)

Amaurose de Leber, v. Albinisme oculaire

Amblyopie, v. Occlusion amblyopia

Amiodaron, v. Lesions iatrogènes

Anatomie, v. Aplasie

Anatomie; Lehrbuch der topo-graphischen Anatomie, 80 (B)

Angeborene Kalarakt; über eine angeborene Katarakt der Ratte. I. Linsenveränderungen während der ersten 14 Tage, 255 (V)

Angiographie fluorescéinique, v. Affections carotidiennes

Angle camérulaire, v. Glaucomato-cyclitic crisis

Angle strabique, v. Schielwinkel-messung 
Anterior chamber, v. Epithel-invasion

Anticorps viraux dans Гuvéí́e, 243 (V)

Aphakie, v. Hydrophile Kontakt-linsen

Aplasie; eine einseitige Aplasie des Nervus opticus, 11

Applanationstonometer; Unter-suchungen des intraokularen Druckes mit dem

Applanationstonometer nach Goldmann, 353 (V)

Aqueous humor, v. Rétinoblastome

Arbeitsmedizinische Fragen in der Ophthalmologie, 415 (B)

Arteriographie, v. Affections carotidiennes

Aspiration und Vorderkammer-irrigation als Hilfe bei der Operation der Cataracta congenita, 292

(V)

Atrophie de Гiris, v. Albinisme oculaire, Mégalocornée

Atrophie par compression; modifications ultrastructurales de la ratine consécutives à une atrophie par compression du nerf optique, $157(\mathrm{~V})$

Augenheilkunde, 78 (B)

Augenkomplikaxionen, v. Coriison-Katarakt, Epiretinale Fibroplasie, Epithelinvasion,

Ethambutol, Largactil, Lesions iatrogènes, Panophthalmilis

Augenmuskeln, v. Esotropies obliques, Myopaíhy, Oculo-myo-dynamometry, Operationsalter

Avian cornea; fine structure of the developing avian cornea, 413 (B)

Bailliart, Paul, 413

Bakteriologie, v. Complications post-

varicelleuses, Mima polymorpha Bande lumineuse, v. Lichtstreifentest Behçet-Uveitis; ERG und Behçet-

Uveitis, 196 (V) Bertalanffy-Methode; die

Berlalanffy-Methode zum Nach-

weis von Tumorzellen im Blut bei

Geschwülsten des Auges, 372 Bibliotheca ophthalmologica, No. 80,

357 (B) Biochemistry of the eye, 357 (B) Blutung, v. Dobésilate de calcium,

Drusenpapille Book reviews, 78, 356, 413 Brachio-okulare Zirkulationszeit,

v. Affections carotidiennes Buchbesprechungen, 78, 356, 413

Capillary resistence, v. Dobésilate

de calcium Carotis, v. Affections carotidiennes Cartographic deformations; the cartographic

deformations of the visual field, 38 Caruncula lacrimalis, v. Oncocytoma Cataract, v.

Angeborene Katarakt, Aspiration, Cortison-Katarakt, Fistulierende Operation, Kryo-extraktion,

Lichtstreifentest, Sehschärfenbestimmung Cataracte secondaire, v. Aspiration Cauterisation, v.

Dystichiasis Chamber angle, v. Glaucomato-

cyclitic crisis Chambre antérieure, v. Epithelinvasion Champ visuel, v. Akkommodations-

phosphen, Behçet-Uveitis, Carío-graphic deformations, Unter-suchungsergebnisse

536

Index rerum

ad Vol. 161

Chirurgie, v. Aspiration, Dakryo-stenose, Dystichiasis, Epithel-invasion, Esotropies obliques,

Fistulierende Operation, Kryo-extraktion, Myopathy, Oculo-myo-dynamometry, Operationsalter, Panophthalmitis, Silberklemme

Chromosomale Anomalie, v. Turner-Syndrom 
Ciliary muscle, v. Akkommodations-phosphen

Circulation, v. Affections caroti-diennes

Coagulation à la lumière, v. Photo-koagulationsbehandlung

Coding system for disorders of the eye. 79 (B)

Coloration, v. Photoreceptor

Coloured stimuli, v. Farbige Licht-reize

Complications oculaires, v. Cortison-Katarakt, Epiretinale Fibroplasie, Epithelinvasion,

Ethambutol, Largactil, Lesions iatrogènes, Panophthalmitis

Complications post-varicelleuses; Complications oculaires graves post-varicelleuses chez

Tadulte, 108 (V)

Congrès, v. Société Suisse d'Ophtal-mologie

Contact lenses, v. Hydrophile Kontaktlinsen, Lentilles cornéennes

Cornea, v. Cortison-Katarakt, Dégénérescence nodulaire, Descemet'sche Membran, Kälte-

applikation, Largactil, Lentilles cornéennes, Lesions iatrogènes, Mégalocornée, Mima

polymorpha, Polyarthritis, Vaccin anti-herpétique

Cornea guttata, v. Descemet'sche Membran

Corneal graft, v. Dégénérescence nodulaire, Mima polymorpha

Corps vitré, v. Dobésilate de calcium, Epiretinale Fibroplasie, Netzhaut-atrophie

Cortex visuel, v. Farbige Lichtreize, 'Visual evoked response'

Cortison-Katarakt; liber Cortison-Katarakt bei Nierentransplan-tation, 125 (V)

Cristallin, v. Angeborene Katarakt, Aspiration, Cortison-Katarakt, Fistulierende Operation,

Hydrophile Kontaktlinsen, Kryo-extraktion, Largactil, Sehschärfen-bestimmung Cryothérapie, v.

Kälteapplikation Cytologie, v. Bertalanffy-Methode

Dakryostenose; Verlaufskontrollen und Spätresultate nach operativer Behandlung praesaccaler

Dakryo-stenosen, 313 (V)

Dégénérescence maculaire, v. Star-gardt's disease, 'Visual evoked response'

Dégénérescence nodulaire; récidive bilatérale d'une dégénérescence nodulaire de Salzmann après greffe perforante de la cornée, 90 (V)

Descemet'sche Membran; gewebliche Diskontinuitätszonen in der ge-sunden und pathologisch ver-änderten menschlichen Descemet-schen Membran, 166 (V)

Developmental glaucoma, v. Glau-comatocyclitic crisis

Diabetes, v. Dobésilate de calcium, Photokoagulationsbehandlung

Díagnostik, v. Affections carotidien-nes, Behçet-Uveitis, Bertalanffy-Methode, Gammagraphie, Nystagmus vertical, Pilocarpintest, Provokationstest, Bétinoblastome, Sehschärfenbestimmung

Dobésilate de calcium; le traitement de la rétinopathie diabétique et de Thémorragie récidivante du vitré par le Dobésilate de calcium, 389

Doxium, v. Dobésilate de calcium

Drusenpapille; zur Frage der Blu-tungen und der venösen Stauung bei der Drusenpapille, 21

Dunkeladaptation, v. Akkommo-dationsphosphen, Behçet-Uveitis

Dynamometrie, v. Affections caro-tidiennes, Oculo-myo-dynamo-metry

Dystichiasis; modifications de

vieilles méthodes opératoires pour la correction de Гentropion et du dystichiasis, 385

Index rerum ad Vol. 161

537

Dystrophie cornéenne, v. Dégé- 
nérescence nodulaire Dystrophie tapéto-rétinienne, $\mathrm{v}$.

Albinisme oculaire, Behçet-Uveitis,

Stargardt's disease

Echographie, v. Gammagraphie,

Mégalocomée EEG, v. Farbige Lichtreize, 'Visual

evoked response' Elektroenzephalogramm, v. Farbige

Lichtreize, 'Visual evoked

response' Elektromyogramm, v. ? > Iyopathy Elektronenmikroskopie, v. Atrophie

par compression, Horizontalzellen,

Oncocytoma, Photoreceptor Elektrookulogramm, v. Nystagmus

vertical, 'Visual evoked response' Elektroretinogramm, v. Albinisme

oculaire, Behçet-Uveitis, Poten-

tiels oscillatoires, 'Visual evoked

response' EMG, v. Myopathy Entropion, v. Dystichiasis EOG, v. Nystagmus vertical, 'Visual evoked response' Epiretinale Fibroplasie und massive

praeretinale Retraktion, 227 (V) Epithelinvasion; Therapie der

Epithelinvasion der Vorderkam-

mer nach Staroperation, 286 (V) Epithelium pigmentaire, v. Aplasie,

Netzhautatrophie ERG, v. Albinisme oculaire, Behçet-Uveitis, Potentiels oscillatoires,

'Visual evoked response' Ergot; the story of ergot, 417 (B) Esotropies obliques; le traitement

chírurgical de choix des esotropies

obliques en V, 340 (V) Ethambutol; la toxicité oculaire de

Гéthambutol, 361 Exophthalmie, v. Exophthalmus,

Gammagraphie Exophthalmus; zur Therapie des

malignen Exophthalmus, 145 (V)

Farbige Lichtreize; über den Einfluss farbigen Lichtreizes auf die visuell evozierten Potentiale

(VEP) des optischen Cortex beim Menschen, 512

Färbung, v. Photoreceptor

Filterkissen, v. Fistulierende Operation

Fistulierende Operation; zur

Kataraktoperation nach fistulie-render Operation, 304 (V)

Fixation, v. Occlusion amblyopia

Fixation instable, v. Nystagmus vertical

Fluorescein angiography of the retina, 358 (B)

Fluorescence photography of the eye, 417 (B)

Fluoreszenzangiographie, v. Affections carotidiennes

Fundus flavimaculatus, v. Stargardt's disease

Gammagraphie; diagnostic des tumeurs intraoculaires et orbitaires par echographie et par

gammagraphie, $132(\mathrm{~V})$

Gefässe, v. Affections carotidiennes, Aplasie, Dobésilate de calcium, Drusenpapille, Epiretinale

Fibroplasie, Glaucomatocyclitic crisis, Photokoagulationsbehandlung

Genetics, v. Albinisme oculaire, Dégénérescence nodulaire, Stargardt's disease

Geschichte der Medizin, v. Sichel Julius

Geschichte der Medizin und Biologie; Basler Veröffentlichungen zur Geschichte der Medizin

und Biologie; Vol. 25: H. Schiess, 79 (B) 
Gesichtsfeld, v. Akkomodations-phosphen, Behçet-Uveitis, Carto graphic deformations, Untersuchungsergebnisse

Glaskörper, v. Dobésilate de calcium, Epiretinale Fibroplasie, Netzhautatrophie

Glaucoma, v. Acéclidine, Appla-nationstonometer, Fistulierende Operation, Pilocarpintest,

Provo-kationstest

Glaucoma juvenile, v. Turner-Syndrom

Glaucomatocyclitic crisis; developmental factor in the etiopatho-genesis of glaucomatocyclitic crisis, 446

538

Index rerum

ad Vol. 161

Greffe cornéenne, v. Dégénérescence nodulaire, Mima polymorpha

Haemorrhagie, v. Dobésilate de calcium, Drusenpapille

Hassal-Henle'sche Warze, v. Desce-met'sche Membran

Haut und Auge, 414 (B)

Hérédité, v. Albinisme oculaire, Dégénérescence nodulaire, Star-gardt's disease

Herpes corneae, v. Kälteapplikatio@, Vaccin antiherpétique

Histochemie, v. Bertalanffy-Methode

Histoire de la médecine, v. Sichel Julius

Histologie, v. Angeborene Kataraki, Aplasie, Atrophie par compression, Dégénérescence

nodulaire, Descemet'sche Membran, Myo-pathy, Oncocytoma, Panophthal-mitis,

Rétinoblastome

Horizontalzellen; die Ultrastruktur der retinalen Horizontalzellen des Menschen, 152 (V)

Hornhaut, v. Cortison-Katarakt, Dégénérescence nodulaire, Descemet'sche Membran,

Kälteappli-kation, Largactil, Lentilles cor-néennes, Lesions iatrogènes, Mégalocornée, Mima

polymorpha, Polyarthritis, Vaccin antiherpétique

Humeur aqueuse, v. Rétinoblastome

Hyalozyten, v. Epiretinale Fibro-plasie

Hydrophile Kontaktlinsen; klinische Erfahrungen mit hydrophilen Kontaktlinsen bei einseitig aphaken Kindern, 279 (V)

Hyperthyreose, v. Exophthalmus

Hypopyon, v. Rétinoblastome

Iatrogene Schäden, v. Cortison-Katarakt, Ethambutol, Largactil, Lesions iatrogènes

Immunologie, v. Polyarthritis

Infektion, v. Anticorps viraux, Complications post-varicelleuses, Mima polymorpha, Panophthal-

mitis

Instrument, v. Applanationstono-meter, Aspiration, Kryoextraktiou,

Oculo-myo-dynamometry, Silber-

klem $\pi$ ic Intraokulardruck, v. Acéclidine,

Applanationstonometer, Püocar-

pintest, Provokationstest Intravitalfärbung am Augenhinter-

grund mit Fluoreszein-Natrium,

78 (B) Iris, v. Albinisme oculaire, Fistulie-

rende Operation, Glaucoma-

tocyclitic crisis, Mégalocornée 
Jod-Abrasio, v. Kälteapplikation

Kälteapplikation; vergleichende Un-tersuchungen über die Wirksam-keit der Kälteapplikation und der Jod-Abrasio in Kombination mit unspezifischer und virostatischer Nachbehandlung bei der Keratitis dendritica, 484

Kammerwasser, v. Rétinoblastome

Kammerwinkel, v. G1/8ucomaío-cycïitic crisis

Kaninchen, v. Epiretinale Fibro-plasie

Kapillarresistenz, v. Dobésüate de calcium

Katarakt, v. Angeborene Katarakt, Aspiration, Cortison-Katarakt, Fistulierende Operation, Kryoextraktion, Lichtstreifentest, Seh-schärfenbestimmung

Kauterisation, v. Dystichiasis

Keratitis, v. Polyarthritis

Keratitis herpetica, v. Kälteapplikation, Vaccin antiherpétique

Keratoplastik, v. Dégénérescence nodulaire, Mima polymorpha

Kontaktlinsen, v. Hydrophile Kontaktlinsen, Lentilles cor-néennes

Kryoextraktion der Linse ohne Assistenz, 300 (V)

Kryotherapie, v. Kälteapplikation

Lacodacryocystomie, v. Dakryo-

stenose Lacoductostomie, v. Dakryostenose Lapin, v. Epiretinale Fibroplasie Largactil; über

Augenveränderungen

bei Langzeitbehandlung mit

Largactil, 118 (V)

rum ad Vol. $161 \quad 539$

Index rei

Laserkoagulation, v. Photokoagu-lationsbehandlung

L.eber'sche Amaurose, v. Albinisme oculaire

Lens, v. Angeborene Katarakt, Aspiration, Cortison-Katarakt, Fistulierende Operation, Hydrophile Kontaktlinsen, Kryo-extraktion, L·argactil, Sehschärfen-bestimmung

Lentilles cornéennes, v. Hydrophile Kontaktlinsen

Lentilles cornéennes; nos experiences avec deux types nouveaux de lentilles cornéennes, 274 (V)

Lesions iatrogènes; lesions oculaires iatrogènes; Faction d'un nouveau medicament contre

Tangine de poitrine, 115 (V)

Letter to the Editors, 418

Lichtkoagulation, v. Photokoagula-tionsbehandlung

Lichtstreifentest; die Verwendung des Lichtstreifentestes bei Vor-liegen dichter

Medient ГÜbungen, 504

Lid, v. Dystichiasis

Light, colour and vision, 356 (B)

Linse, v. Angeborene Katarakt, Aspiration, Cortison-Katarakt, Fistulierende Operation,

Hydrophile Kontaktlinsen, Kryo-extraktion, L·argactil, Sehschärfen-bestimmung

Lipoprotein membrane, v. Photo-receptor

Livres nouveaux, 78, 356, 413

Macular degeneration, v. Stargardt's disease, 'Visual evoked response'

Makulaloch, v. Silberklemme

Malformation, v. Aplasie 
Marchesani-Sydrom, v. Megalocornee

Medientrübungen, v. Lichtstreifentest

Medikament, v. Acéclidine, Cortison-Katarakt, Dobésilate de calcium, Ethambutol,

Kälteapplikation, L·argactil, Lesions iatrogènes, Vaccin antiherpétique

Megalocornee; syndrome de Marche-sani associé à une megalocornee et à une atrophie de Гiris;

etude échographique, 264 (V)

Mélanose oculaire congénitalc bénigne, 245 (V)

Methode, v. Aspiration, Bertaianffy-Methode, Dakryostenose, Dystichiasis, Esotropies obliques,

Fistulierende Operation, Kryoextrak-tion, Lichtstreifentest, Oculo-myo-dynamometry,

Operationsalter, Photoreceptor, Schielwinkel-messung, Silberklemme

Microscopie électronique, v. Atrophie par compression, Horizontalzellen, Oncocytoma,

Photoreceptor

Mikroaneurysma, v. Gláucoma-tocyclitic crisis, Photokoagula-tivnsbehandlung

Mikrophthalmie, v. Aplasie

Mima polymorpha; Ulcus corneae durch Mima polymorpha, 98 (V)

Missbildung, v. Aplasie

Multiple Sklerose, v. Nystagmus vertical

Muscles oculaires, v. Esotropies obliques, Myopathy, Oculo-myo-dynamometry, Operationsalter

Mykosis, v. Panophthalmitis

Myopathy; surgery for ptosis and strabismus in ocular myopathy, 477

Nachruf: Paul Bailliart, 413

Nachstar, v. Aspiration

Nécrologe: Paul Bailliart, 413

Nerf optique, v. Aplasie, Atrophie par compression, Ethambutol

Netzhaut, v. Akkommodations-phosphen, Albinisme oculaire, Aplasie, Atrophie par

compression, Dobésilate de calcium, Epiretinale Fibroplasie, Horizontalzellen, Netzhautatrophie, Panophthalmitis, Photokoagulationsbehand-lung, Photoreceptor, Potentiels oscillatoires,

Sehschärfenbestim-mung, Silberklemme, Stargardt's disease, 'Visual evoked response'

Netzhautablösung, v. Akkommoda-tionsphosphen, Netzhautatrophie, Silberklemme

Netzhautatrophie; periphere gürtel-förmige Netzhautatrophie bei Ablatio retinae, 217 (V)

Neuritis optica, v. Ethambutol

540

Index rerum

ad Vol. 161

Neurologie; Elemente der Neuro-iogie auf der Grundlage von Physiologie und Klinik, 359 (B)

Neuro-Ophthalmologie, v. Affections carotidiennes, Nystagmus vertical

Nierentransplantation, v. Cortison-Katarakt

Nystagmus vertical; fixation instable et nystagmus vertical, 31

Obituary: Paul Bailliart, 413

Occlusion amblyopia, 55

Occupational and medicative

hazards in ophthalmology, 414 (B)

Ocular fundus; manual and color atlas of the ocular fundus, 80 (B)

Ocular muscles, v. Esotropies obliques, Myopathy, Oculo-myo-dynamometry, Operationsalter

Oculo-myo-dynamometry, the strength and work of extraocular muscles in squint, 491 
Oncocytoma; ultrastructure of the caruncular oncocytoma, 63

Open angle glaucoma, v. Acéclidine, Pilocarpintest

Operation, v. Aspiration, Dakryo-stenose, Dystichiasis, Epithel-invasion, Esotropies obliques,

Fistulierende Operation, Kryo-extraktion, Myopathy, Oculo-myo-dynamometry, Operationsalter, Panophthalmitis, Silberklemme

Operationsalter; über das günstigste Operationsalter bei Strabismus convergens und

sursoadductorius. Beidseitige Bücklagerung des M. rectus int. bzw. Myektomie des M. obi. inf., 466

Operative surgery, 80 (B)

Ophthalmodynamometrie, v. Affections carotidiennes

Ophthalmology, 359 (B)

Optic cortex, v. Farbige L·ichtreize, 'Visual evoked response'

Optic disc, v. Aplasie, Drusenpapille, Ethambutol

Opticus, v. Aplasie, Atrophie par compression, Ethambutol

Oto-rhino-laryngology; advances in oto-rhino-laryngology, 357 (B)

Outflow resistance, v. Acéclidine, Pilocarpintest

Paedo-Ophthalmologie, v. Hydro-phile Kontaktlinsen, Operations-alter

Panophthalmitis; mykotische Panophthalmitis nach Netzhaut-operation, 237 (V)

Papule, v. Aplasie, Drusenpapille, Ethambutol

para-Fluorphenylalanin, v. Kälte-applikation

Paupière, v. Dystichiasis

Perimetrie, v. Akkommodations-phosphen, Behçet-Uveitis, Cartographic deformations, Unter-

suchungsergebnisse

Phosphène accommodatif, v. Akkommodationsphosphen

Photographische Messung, v. Schiel-winkelmessung

Photokoagulationsbehandlung; zur Photokoagulationsbehandlung der diabetischen Retinopathie, $219(\mathrm{~V})$

Photoreceptor; polarization optical studies on the ultrastructure of the photoreceptor, 402

Pigmentation, v. Albinisme oculaire, Mélanose

Pigmentepithel, v. Aplasie, Netz-hautatrophie

Pilocarpintest zur Glaukom-diagnostik mit Hilfe der Tono-graphie, 451

Pilzinfektion, v. Panophthalmitis

Polarisations-Mikroskopie, v. Photoreceptor

Polyarthritis; Hornhauterkrankun-gen bei chronischer Polyarthritis, 437

Ponction camérulaire, v. Rétino-blastome

Potentiels oscilîatoires; essai de standardisation d'une méthode pour $\Gamma$ étude des potentiels

oscilîatoires chez Thomme. I. Elude morphologique, 202 (V)

Praeretinale Retraktion, v. Epireti-nale Fibroplasie

Praesaccal dacryostenosis, v. Dakryostenose

Precis d'ophtalmologie, 356 (B)

Pression oculaire, v. Acéclidine, Applanationstonometer, Pilocarpintest, Provokatíonstest Index rerum

ad Vol. 161

541

Problèmes actuels en ophtalmologie, 
415 (B) Proceedings, v. Schweizerische

Ophthalmologische Gesellschaft Provokationstest; über die Reak-

tionsdifferenz von Normal- und

Glaukomaugen auf Provokations-

tests, 460 Ptosis, v. Myopathy Ptosis, 80 (B)

Rabbit, v. Epiretinale Fibroplasie Rat, v. Angeborene Katarakt Ratte, v. Angeborene Katarakt

Resistance à Гécoulement, v. Acé-

clidine, Pilocarpintest Resistance capillaire, v. Dobésilate

de calcium Retina, v. Akkommodations-phosphen, Albinisme oculaire, Aplasie, Atrophie par

compression, Dobésilate de calcium, Epiretinale Fibroplasie, Horizontalzellen, Netzhautatrophie,

Panophthalmitis, Photokoagulationsbehand-lung, Photoreceptor, Potentiels oscillatoires,

Sehschärfenbestim-mung, Silberklemme, Stargardt's disease, 'Visual evoked response' Retinitis pigmentosa, v. Albinisme

oculaire Rétinoblastome et hypopyon

(epilogue), 139 (V) Rheumatoide Arthritis, v. Polyarthritis

Salzmann'sche Hornhautdegene-ration, v. Dégénérescence nodulaire

Schädelhirnverletzung; die Augen-symptomatik bei der frischen Schädelhirnverletzung, 356 (B)

Schielen, v. Esotropies obliques, Occlusion amblyopia, Oculo-myo-dynamometry, Myopathy,

Opera-tionsalter, Schielwinkelmessung

Schielwinkelmessung; neue photo-graphische Methode der Schielwinkelmessung, 346 (V)

Schilddrüse; die Schilddrüse, 413 (B)

Schweizerische Ophthalmologische Gesellschaft, 62. Generalversamm-lung, Biel, 1969, 83-355

(V)

Sclérose multiloculaire, v. Nystagmus vertical

Sehschärfe, v. Lichtstreifentest, Sehschärfenbestimmung

Sehschärfenbestimmung; retinaie Sehschärfenbestimmung bei Katarakt, 175 (V)

Sehübungen, 80 (B)

Septicémie, v. Complications post-varicelleuses

Sichel, Julius; Julius Sichel aus Frankfurt (1802-1868), ein Pionier der modernen französischen

Augenheilkunde, 83 (V)

Silberklemme; Erfahrungen mit der Silberklemme bei Makulaloch-bedingten

Netzhautablösungen, 210 (V)

Sklera, v. Mélanose, Panophthalmitis

Skleritis necroticans, v. Polyarthritis

Société Suisse d'Ophlalmologie, 62e Assemblée generate, Bienne 1969, 83-355 (V)

Squint, v. Esotropies obliques, Occlusion amblyopia, Oculo-myo-dynamometry, Myopathy,

Opera-tionsalter, Schielwinkelmessung

Staining, v. Photoreceptor

Stargardt's disease; abnormal findings in relatives of patients with juvenile hereditary macular degeneration (Stargardt's disease), 1

Stimulation à la couleur, v. Farbige Lichtreize

Strabismus, v. Esotropies obliques, Occlusion amblyopia, Oculo-myo-dynamometry, Myopathy, Opera-tionsalter, Schielwinkelmessung

Surgery, v. Aspiration, Dakryo-stenose, Dystichiasis, Epithel-invasion, Esotropies obliques,

Fistulierende Operation, Kryo-extraktion, Myopathy, Oculo-myo-dynamometry, Operationsalter, Panophthalmitis, Silberklemme 
Symptom; vom Symptom zur Diagnose, 358 (B)

Syndrom, v. Mégalocornée, Turner-Syndrom

Tapetretinal dystrophy, v. Albinisme oculaire, Behçet-Uveitis, Stargardt's disease

542

Index Гerum

ad Vol. 161

Tension oculaire, v. Acéclidine, Applanationstonometer, Pilo-carpintest, Provokationstest

Therapeutics; handbook of ocular therapeutics and pharmacology, 416 (B)

Therapie, v. Acéclidine, Aspiration, Cortison-Katarakt, Dobésilate de calcium, Epithelinvasion,

Exoph-thalmus, Kälteapplikation, Largac-til, Lesions iatrogènes, Myopathy, Operationsalter,

Photokoagula-tionsbehandlung, Vaccin anti-herpétique

Thyreostatica, v. Exophthalmus

Tonographie, v. Acéclidine, Pilo-carpintest

Tonometrie, v. Applanationstonometer

Topooptical staining method, v. Photoreceptor

Toxicité, v. Ethambutol, Largactil, Lesions iatrogènes

Transplantation, v. Cortison-Katarakt

Tuberculose, v. Ethambutol

Tumeurs; nomenclature illustrée des tumeurs, 359 (B)

Tumor, v. Atrophie par compression, Bertalanffy-Methode, Gamma-graphie, Nystagmus

vertical, Oncocytoma, Rétinoblastome

Turner-Syndrom; Glaucoma juvenile beim Turner-Syndrom, 264 (V)

Ulcus comeae, v. Mima polymorpha, Polyarthritis

Untersuchungsergebnisse; Streuun-gen perimetrischer Untersuchungsergebnisse, 180 (V)

Uveitis, v. Anticorps viraux, Behçet-Uveitis

Vaccin antiherpétique; erste persön-liche Erfahrungen mit dem vaccin antiherpétique 'Diamant', $104(\mathrm{~V})$

Vaisseaux, v. Affections carotidien-nes, Aplasie, Dobésilate de calcium, Drusenpapille,

Epiretinale Fibroplasie, Glaucomatocyclític crisis, Photokoagulationsbehand-lung

Varia, 360, 422

Varicelle, v. Complications post-varicelleuses

Vererbung, v. Albinisme oculaire, Dégénérescence nodulaire, Stargardt's disease

Verhandlungsberichte, v. Schweize-rische Ophthalmologische Gesell-schaft

Vessels, v. Affections carotidiennes, Aplasie, Dobésilate de calcium, Drusenpapille, Epiretinale

Fibroplasie, Glaucomatocyclitic crisis, Photokoagulationsbehandlung

Virus, v. Anticorps viraux, Complications post-varicelleuses, Kälteapplikation, Mima

polymorpha, Vaccin antiherpétique

'Visual evoked response' im opli-schen Cortex bei Makuladegene-rationen, 187 (V)

Visual field, v. Akkommodations-phosphen, Behçet-Uveitis, Carlo-graphic deformations,

Untersuchungsergebnisse

Visuell evoziertes Potential, v. Far-bige Lichtreize, 'Visual evoked response'

Visus, v. Lichtstreifentest, Sehschär-fenbestimmung

Vitreous; the vitreous in clinical ophthalmology, 416 (B)

Vitreous body, v. Dobésilate de calcium, Epiretinale Fibroplasie, Netzhautatrophie

Vogt'scher Ring, v. Albinisme oculaire 
Vorderkammer, v. Epithelinvasion

Vorderkammerirrigitation, v. Aspiration

Vorderkammer-Punktion, v. Rétinoblastome

V-Syndrom, v. Esotropies obliques

Wegener'sche Granulomatose,

v. Polyarthritis Weitwinkelglaukom, v. Acéclidine,

Pilocarpintest

Ziliarmuskel, v. Akkommodations-phosphen

Z. Zirkulation

v. Affections carotidiennes

Zytologie

v. Bertalanffy-Methode 